University of Nebraska - Lincoln

DigitalCommons@University of Nebraska - Lincoln

6-1994

\title{
Photoconductivity and grating response time of a photorefractive polymer
}

\author{
Brian E. Jones \\ University of Nebraska-Lincoln \\ Stephen Ducharme \\ University of Nebraska-Lincoln, sducharme1@unl.edu \\ Martin M Liphardt \\ University of Nebraska - Lincoln \\ Arosha Goonesekera \\ University of Nebraska-Lincoln \\ James M. Takacs \\ University of Nebraska-Lincoln, jtakacs1@unl.edu \\ See next page for additional authors
}

Follow this and additional works at: https://digitalcommons.unl.edu/physicsducharme

Part of the Physics Commons

Jones, Brian E.; Ducharme, Stephen; Liphardt, Martin M; Goonesekera, Arosha; Takacs, James M.; Zhang, Lei; and Athalye, Rajan, "Photoconductivity and grating response time of a photorefractive polymer" (1994). Stephen Ducharme Publications. 78.

https://digitalcommons.unl.edu/physicsducharme/78

This Article is brought to you for free and open access by the Research Papers in Physics and Astronomy at DigitalCommons@University of Nebraska - Lincoln. It has been accepted for inclusion in Stephen Ducharme Publications by an authorized administrator of DigitalCommons@University of Nebraska - Lincoln. 


\section{Authors}

Brian E. Jones, Stephen Ducharme, Martin M Liphardt, Arosha Goonesekera, James M. Takacs, Lei Zhang, and Rajan Athalye 


\title{
Photoconductivity and grating response time of a photorefractive polymer
}

\author{
Brian E. Jones, Stephen Ducharme, Martin Liphardt, and Arosha Goonesekera \\ Department of Physics and Astronomy, Center for Materials Research and Analysis, \\ University of Nebraska, Lincoln, Nebraska 68588-0111
}

James M. Takacs, Lei Zhang, and Rajan Athalye

Department of Chemistry, Center for Materials Research and Analysis, University of Nebraska, Lincoln, Nebraska 68588-0111

Received August 19, 1993; revised manuscript received January 3, 1994

\begin{abstract}
We report the photoconductive properties and photorefractive grating response time of a polymer mixture composed of 40-wt. \% dissolved diethylamino-benzaldehyde diphenyl hydrazone (DEH) and the non-cross-linking epoxy polymer Bisphenol A 4,4'-nitroaminostilbene. The films have improved photoconductive sensitivities as high as $2.1 \times 10^{-10} \mathrm{~cm} /(\mathrm{W} \Omega)$ at a wavelength of $650 \mathrm{~nm}$ with a corresponding reduction of the grating response time constant to $0.11 \pm 0.02 \mathrm{~s}$ at an intensity of $1 \mathrm{~W} / \mathrm{cm}^{2}$. The nitro-aminostilbene chromophore is deduced to be the source of photogenerated charge carriers on the basis of a comparison of the wavelength dependence of the photoconductivity and absorption coefficient. Degradation of the photoconductivity and the dark conductivity as well as of the photorefractive speed with sample age is attributed to precipitation of the DEH; this explanation is supported by x-ray diffraction observations of crystal growth in the polymer.
\end{abstract}

\section{INTRODUCTION}

The photorefractive effect is a persistent but reversible change in the refractive index of an electro-optic material caused by nonuniform illumination. It was first observed as detrimental optical damage in lithium niobate and other crystals used for second-harmonic generation. ${ }^{1}$ The photorefractive effect occurs in materials that have these three properties ${ }^{2}$ : a linear electro-optic (Pockels) response, photoconduction, and photosensitive traps. The photorefractive effect is the basis of numerous proposed applications, including integrated optics, optical processing, optical data storage, optical computing, communications, image processing, optical switching, thresholding, laser resonators, simulation of neural networks, and studies of nonlinear dynamics. Until recently all photorefractive materials have been inorganic crystals.

Promising new photorefractive materials made of electro-optic polymers mixed with charge-transport agents and photosensitizers have been reported recently. ${ }^{3-7}$ The nonlinear-optical polymers ${ }^{8,9}$ provide structure and cohesiveness as well as the necessary electro-optic response. The photosensitizers (sometimes the host electro-optic polymer acts as the photosensitizer) are the source of photocarriers, which are carried by the charge-transport agents. ${ }^{10,11}$ Recent research in photoconducting polymers provides an excellent model of the production of a composite system whose individual components work together to produce a complex desired behavior.

Photorefractive polymers may replace crystals in photorefractive applications because of the polymers' relative low cost and ease of fabrication into a variety of bulk and thin-film devices. They are readily altered to conform to complex device requirements imposed by environment, size, shape, physical flexibility, reliability, and other constraints. Polymers are also amenable to mass production in a variety of forms and shapes; they can be cast, spun, painted, molded, injected, or extruded, and they can be patterned by common lithographic techniques. Specifically, the new photorefractive polymers are ideally suited for application in integrated optoelectronics by virtue of their low cost, by their ease of processing and patterning into waveguide devices, and by the all-optical nature of the photorefractive interaction (electronic control is also possible). A model system integrated on one chip or subassembly may contain electronic devices, laser or LED sources, optical processing devices, and detectors. In integrated optoelectronics, photorefractive materials can perform moderate-speed operations such as switching, modulation, thresholding, multiplexing and demultiplexing, and reconfiguration. Photorefractive polymer films or device arrays used in free-space transmission can provide large-aperture parallel processing or storage capability for, e.g., image processing or display technology that is easily and economically fabricated.

Polymers have potentially higher photorefractive figures of merit than crystals. It is necessary, however, to improve the performance of photorefractive polymers before they are suitable for commercial application. Improvements in polymer composition and in sample fabrication have already yielded photorefractive polymers with photorefractive coupling gains ${ }^{12,13}$ as high as $56 \mathrm{~cm}^{-1}$ and photorefractive response times faster than $0.1 \mathrm{~s}^{12-15}$ with moderate optical intensity $\left(1 \mathrm{~W} / \mathrm{cm}^{2}\right)$. This performance is competitive with existing photorefractive crystals. Photorefractive and other nonlinear-optical polymers will retain many of the drawbacks, as well as 
the advantages, of polymers in general, particularly their relative intolerance to elevated temperatures and the tendency of the artificially induced molecular alignment to relax at ambient temperatures-a consequence of the nonequilibrium state. Removing or reducing these difficulties in nonlinear-optical polymers is an area of intense current research worldwide, and a number of successful approaches have been demonstrated. ${ }^{16-18}$

We compare the photorefractive speed and the photoconductivity of a new polymer that has improved photorefractive sensitivity and can be poled. The material is a mixture of the electro-optic polymer bisphenol A 4,4'-nitroaminostilbene (bisA-NAS) and 40-wt. $\%$ diethylamino-benzaldehyde diphenyl hydrazone (DEH), ${ }^{11}$ a hole-transport agent incorporated to carry holes photogenerated from the nitroaminostilbene (NAS) chromophore. ${ }^{3}$ The polymer exhibits the necessary electro-optic and photoconductive responses and unique features characteristic of photorefractive grating formation, readout, and erasure. The photocurrent is approximately proportional to the applied field and to the optical power. The fatigue of the photocurrent is consistent with the presence of at least two distinct types of active trap. ${ }^{6}$ Type A traps are highly photosensitive and are associated with the electro-optic chromophore NAS. Type B traps, of unknown origin, have low photogeneration efficiencies and collect charges liberated from the type A traps. Photorefractive response time is observed to decrease concurrent with the degrading photoconductivity over $400 \mathrm{~h}$. This degradation is attributed to DEH precipitation, a hypothesis supported by a similar degradation in the dark conductivity and the appearance of crystalline DEH x-ray diffraction peaks in aged samples.

\section{SYNTHESIS, FABRICATION, AND CHARACTERIZATION}

The bisA-NAS polymer (Fig. 1) was synthesized by the method used to synthesize bisphenol A 4,4'-nitroaminotolane (bisA-NAT). ${ }^{6}$ Samples were fabricated with 40-wt. \% $\mathrm{DEH}^{11}$ as described previously. ${ }^{3,6}$ The samples were $145 \mu \mathrm{m}$ thick, showed slight optical scattering that was due to inhomogeneity, and had absorption coefficients of $60 \pm 5 \mathrm{~cm}^{-1}$ at $650 \mathrm{~nm}$ (Fig. 2). The dielectric constant of the samples was $3.6 \pm 0.4$ at $1 \mathrm{kHz}$, and the (reversible) dielectric strength of the samples was at least $120 \mathrm{kV} / \mathrm{cm}$.

The photorefractive polymer samples were prepared as follows: First, two $25 \mathrm{~mm} \times 12 \mathrm{~mm} \times 1.1 \mathrm{~mm}$ indium tin oxide (ITO) -coated soda lime glass plates provided by Applied Films Laboratories, Inc., were cut and prepared by the etching of an electrode pattern in the ITO coating (Fig. 3). The desired electrode area was first covered with tape. Then the glass plates were etched, for approximately $20 \mathrm{~min}$, in a mixture of 3 parts nitric acid and 1 part hydrochloric acid, which was diluted 1 to 1 with water. The two conducting surfaces of the slides were cleaned with methanol followed by acetone. The slides were placed, conducting surface up, onto a hot plate controlled by an Omega temperature controller and platinum thermometer. The temperatures of the top surfaces of the slides were maintained at $95 \pm 0.5^{\circ} \mathrm{C}$. Second, the electro-optic polymer bisA-NAS was dissolved in 1- methoxy-2-propanol at a concentration of $25 \mathrm{mg}$ of polymer to $1 \mathrm{~mL}$ of solvent. The hole-transport agent $\mathrm{DEH}$ was dissolved in the same solution at $16.7 \mathrm{mg}$ per $1 \mathrm{~mL}$ of solvent. The solution was then filtered to $2 \mu \mathrm{m}$. Third, approximately $1 \mathrm{~mL}$ of the polymer-DEH solution was dripped alternately onto the ITO electrodes etched on each of the slides [Fig. 3(a)]. The time between drips allowed the solvent to evaporate sufficiently to prevent overflow. The slides remained on the hot plate for 30 min after the dripping ceased to allow most of the solvent to evaporate. Fourth, two small glass spacers, $15 \mathrm{~mm} \times 1 \mathrm{~mm} \times 145 \mu \mathrm{m}$ (cut from microscope cover slips) were placed on either side of the polymer on one of the glass slides as shown in Fig. 3(b). The second slide was placed polymer side down onto the side with the spacers. The slides were arranged so that the polymer was sandwiched between the ITO electrodes [Fig. 3(b)]. The spacers kept the polymer contained when the two slides were assembled and also maintained uniform thickness throughout the film. The slides were positioned off

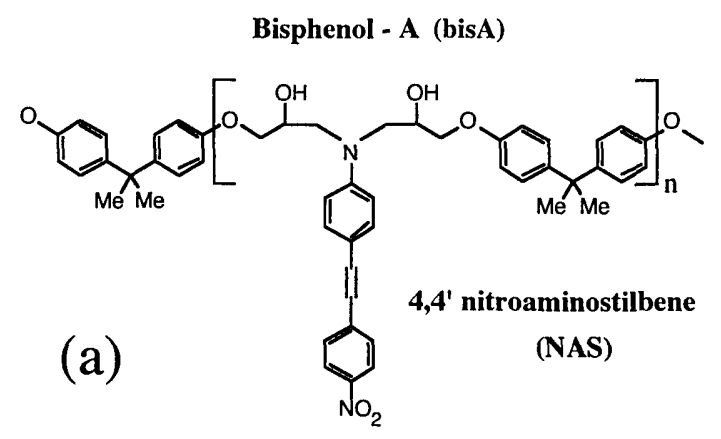

diethylamino-benzaldehyde diphenyl hydrazone

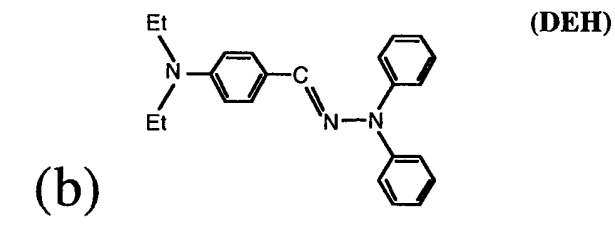

Fig. 1. (a) The electro-optic polymer bisA-NAS. (b) The holetransport agent DEH.

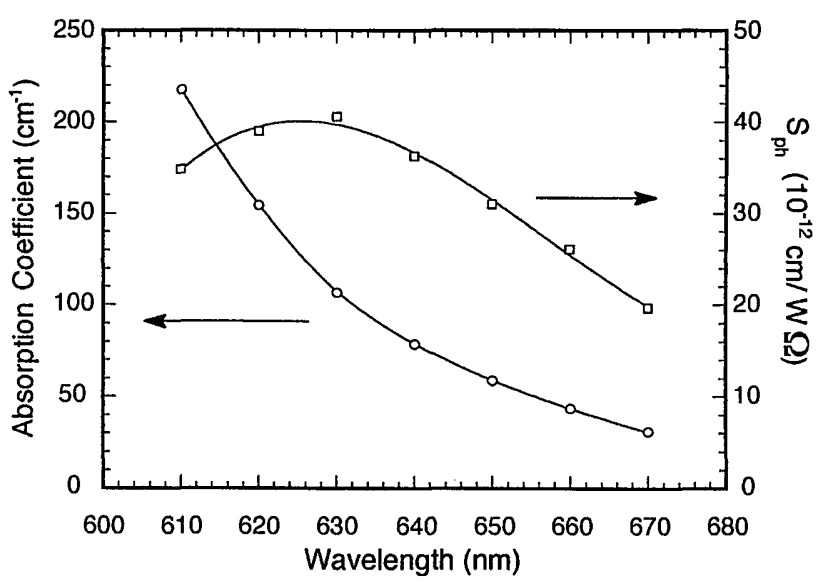

Fig. 2. Photosensitivity ( $S_{\mathrm{ph}}$ ) (squares) and absorption coefficient (circles) versus wavelength of sample 2,17 days after fabrication at $69 \mathrm{kV} / \mathrm{cm}$ and $50 \mathrm{~mW}$. The photoconduction curves do not show fatigue. 

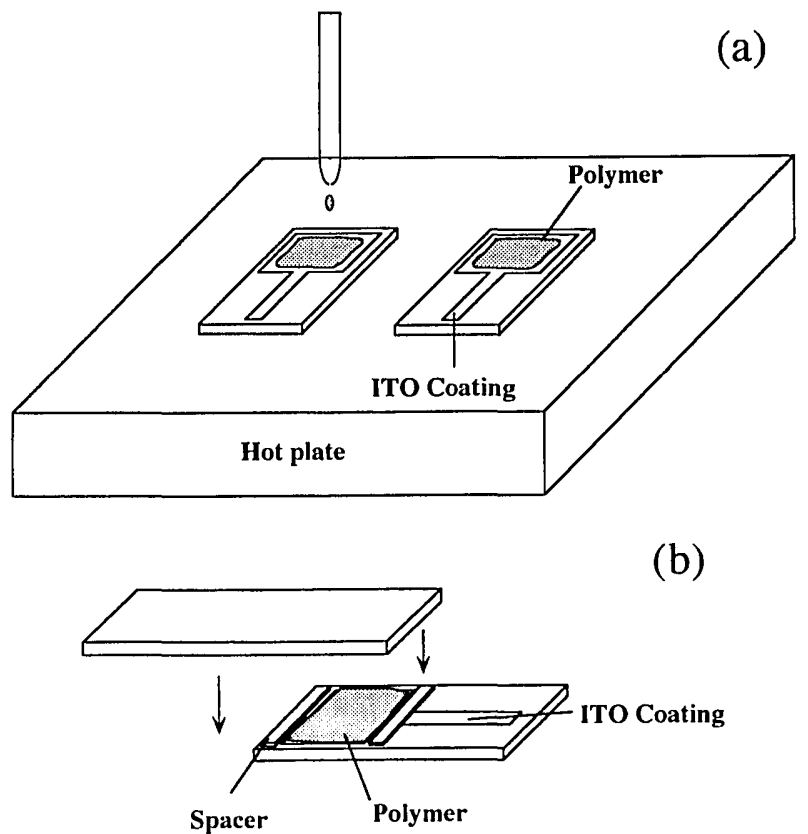

Fig. 3. (a) Procedure for dripping the polymer-DEH solution onto ITO-coated glass plates. (b) Assembly of the photorefractive polymer sample. The active region between the electrodes was $145 \mu \mathrm{m}$ thick and $1 \mathrm{~cm}^{2}$ in area.

center so as to permit access to both electrodes. The assembled sample was removed from the hot plate and allowed to cool and harden for $15 \mathrm{~min}$. The resulting hardened polymer held the sample securely.

The dielectric strength of each sample was estimated by measuring the current through the sample as the voltage was slowly increased. Permanent damage occurred when the arcing through the sample caused a burn and ruined the sample for any future experiments. It was found that any permanent damage caused by excess voltage was preceded by frequent nondamaging spikes in the current at a slightly lower voltage. The maximum safe voltage was defined as the lowest voltage causing an average of two spikes per minute. All experiments with the sample were therefore carried out at or below the maximum safe voltage with no apparent permanent damage. Typical maximum safe voltages for the bisA-NAS:DEH samples were between 1000 and $2000 \mathrm{~V}$, corresponding to an internal electric field of $69-138 \mathrm{kV} / \mathrm{cm}$. All measurements were made at room temperature.

\section{A. Electro-optic Response}

The linear electro-optic (Pockels) response of each sample was measured by a low-frequency $(\leq 1000 \mathrm{~Hz})$ phasemodulation technique with the sample mounted in a Mach-Zehnder interferometer ${ }^{19}$ with a $632.8-\mathrm{nm}, 6-\mathrm{mW}$, $\mathrm{TEM}_{00}$, helium-neon laser. The laser light was $p$ polarized at a $45^{\circ}$ incidence angle (in air), yielding a typical effective electro-optic response $n^{3} r_{\text {eff }}=1 \pm$ $0.5 \mathrm{pm} / \mathrm{V}$ with a $69 \mathrm{kV} / \mathrm{cm}$ alignment electric field in nominally unpoled samples. Piezoelectric, electrostrictivo, and electromechanical contributions have been accounted for by independent measurement in a reflection geometry. ${ }^{19}$ Two samples with glass temperatures $T_{g} \approx 60^{\circ} \mathrm{C}$ were successfully poled..$^{20}$ After the poling, these samples had a zero-field electro-optic response of approximately $1.5 \mathrm{pm} / \mathrm{V}$ at room temperature; the electrooptic response relaxed with a room-temperature half-life of $1100 \mathrm{~h}$ at zero electric field, as is shown in Fig. 4. Application of a $69-\mathrm{kV} / \mathrm{cm}$ electric field to the samples increased the electro-optic response $n^{3} r_{\text {eff }}$ by $0.5-1 \mathrm{pm} / \mathrm{V}$ whether the samples were poled or not. Both samples were discolored, damaged by the poling procedure.

\section{B. Photoconduction}

We measured the conductivity of each sample by applying a dc voltage and measuring the current through the sample (refer to Fig. 5). Each sample was mounted inside a diecast aluminum box on top of a small block of Teflon that was covered with black electrician's tape to minimize diffuse reflection from the Teflon after the beam had passed through the sample. Two small spring contacts made of beryllium-copper attached to the block made electrical connections to the ITO electrodes on the glass slides. One contact was connected to a Stanford Research Systems, Inc., Model PS 350 high-voltage power supply, the other to a Keithley Model 428 current amplifier. The output of the current amplifier was recorded on a Linseis Model L4100 strip-chart recorder,

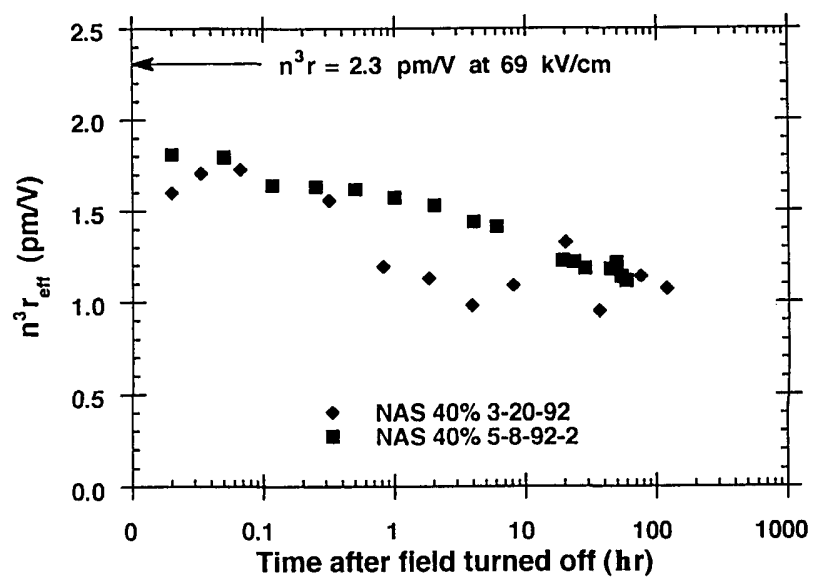

Fig. 4. Relaxation of the electro-optic response for two samples after poling.

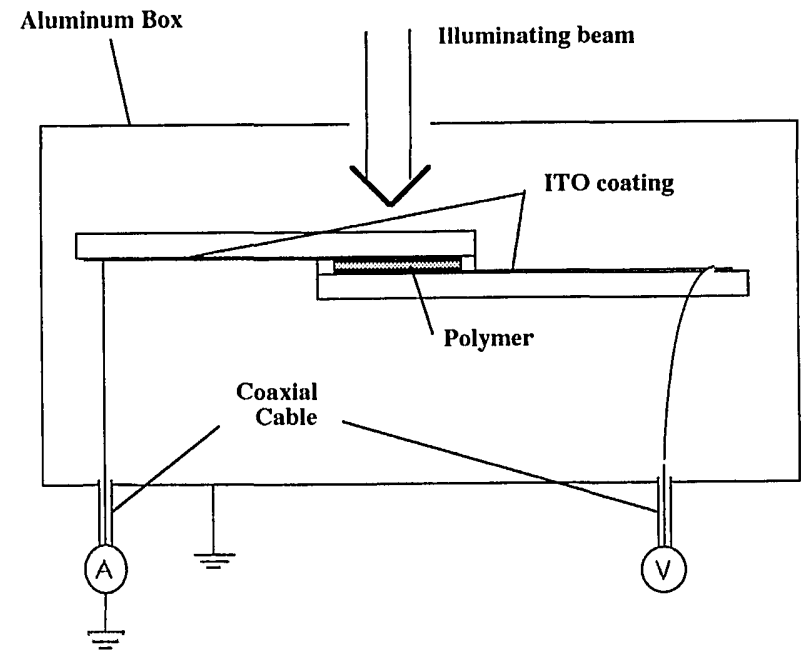

Fig. 5. Experimental arrangement for measurement of the photoconductivity. The sample is illuminated while a constant dc voltage $\mathrm{V}$ is maintained across the electrodes with area $\mathrm{A}$, and the current $J$ through the sample is monitored. 
on a Tektronix 2440 digital oscilloscope, or by computer. The samples were illuminated at normal incidence by a 0.55-mm-diameter (FWHM) beam from a cw dye laser.

We determined the photoconductivity $\sigma_{\mathrm{ph}}$ of the samples by illuminating each with a laser while a constant voltage was maintained across the sample electrodes. The current through the sample was monitored before, during, and after illumination with light of intensity $I$, as shown in Fig. 6 . The rise in current flowing through the sample caused by this light, the photocurrent $\Delta J$, was measured and recorded. The photosensitivity, $S_{\mathrm{ph}}=\sigma_{\mathrm{ph}} / I=L \Delta J / V P$, was calculated from the photocurrent $\Delta J$, applied voltage $V$, the thickness of the sample $L$, and the power of the beam $P$.

Most bisA-NAS:DEH samples exhibited a decrease in photocurrent shortly after illumination, which we call photocurrent fatigue, as is shown in Fig. 6. Sample 4 exhibited this fatigue 1 day after fabrication, whereas sample 5 had no noticeable fatigue for at least 1 month after fabrication. As the figure shows, the initial photocurrent is reduced by $40 \%$ over $\sim 5 \mathrm{~min}$. Most samples had photocurrent fatigue to a final value $J_{f}$ of between $30 \%$ and $75 \%$ of the initial value $J_{i}$. There was no persistence of the photocurrent; the current returned to its original dark value immediately after the illumination ceased. Once photocurrent fatigue had occurred in a sample, the high initial peak in the fatigued curve was not repeated unless the sample was restored by being left for at least an hour in the dark with no applied voltage.

This fatigue is consistent with the presence of two types of trap for photogenerated charge carriers, a model that was proposed to explain photoconduction fatigue and grating competition in bisA-NAT:DEH 40\%. ${ }^{6}$ (Here we discuss the features of this model as they apply to the photoconduction fatigue; the application of this model to photorefractive charge gratings will be discussed below.) The type A traps are the main traps from which charge carriers are easily photogenerated, and the type B traps are shallow, weakly photoionizable traps. The type A traps are presumably a subset of the NAS chromophores. The origin of the type $B$ traps is unknown; they may be additional impurities, polymer-chain defects, or even another subset of the NAS chromophores. In this model the type B traps are initially empty and neutral, but they are responsible for trapping a large fraction of the charge carriers initially freed from the type A traps. Samples were restored presumably when the shallow Type B traps emptied into the Type A traps.

The initial $S_{\mathrm{ph}}$ of each of the samples discussed in this paper is recorded in Table 1 . There were no indications of space-charge buildup or injection in long-term measurements or in field-reversal tests. The possibility that illumination increased the current by heating the sample was tested by illumination of samples with 514.5-nm (strongly absorbed) light. The temperature of the top glass plate increased by no more than $0.1^{\circ} \mathrm{C}$, and the current did not measurably change. Unless otherwise noted, all photosensitivity measurements were made before samples exhibited photocurrent fatigue curves.

The photocurrent was found to be linear in intensity. However, as can be seen in Fig. 7, there was a nonzero $y$ intercept. This suggested a nonlinear relationship at low intensities (below $5 \mathrm{~W} / \mathrm{cm}^{2}$ ). As a result the photo- sensitivity (also in Fig. 7), which is proportional to the photocurrent divided by intensity, was not independent of intensity below approximately $15 \mathrm{~W} / \mathrm{cm}^{2}$. The photosensitivity decreases by a factor of $\sim 2$ between $3.5 \mathrm{~W} / \mathrm{cm}^{2}$ and $15 \mathrm{~W} / \mathrm{cm}^{2}$.

The photosensitivity $\left(S_{\mathrm{ph}}\right)$ increased steadily with the applied field, as is shown in Fig. 8. $S_{\mathrm{ph}}$ increased by a factor of $\sim 2$ from 6.9 to $96.5 \mathrm{kV} / \mathrm{cm}$. Since it was determined that there was no space-charge buildup in the samples, this was likely due to field effects on the photogeneration rate $^{21}$ and carrier mobility. ${ }^{22}$

The photosensitivity of sample 2 was proportional to the absorption coefficient at longer wavelengths $(640-670 \mathrm{~nm})$, when the sample was uniformly illuminated. At shorter wavelengths the photocurrent decreased owing to the increased attenuation of the beam near the surface of the sample. Since the absorption at these wavelengths is entirely due to the NAS chromophore, the NAS chromophore must be the primary source of photogenerated charge carriers.

\section{Degradation of Photoconductivity}

The photoconductivity and dark conductivity of all bisANAS:DEH samples decreased with the age of the sample. As Fig. 9 shows, the photosensitivity and dark conductivity decrease steadily with sample age. It has been proposed that the cause of this degradation is the gradual precipitation of the $\mathrm{DEH}$ molecule

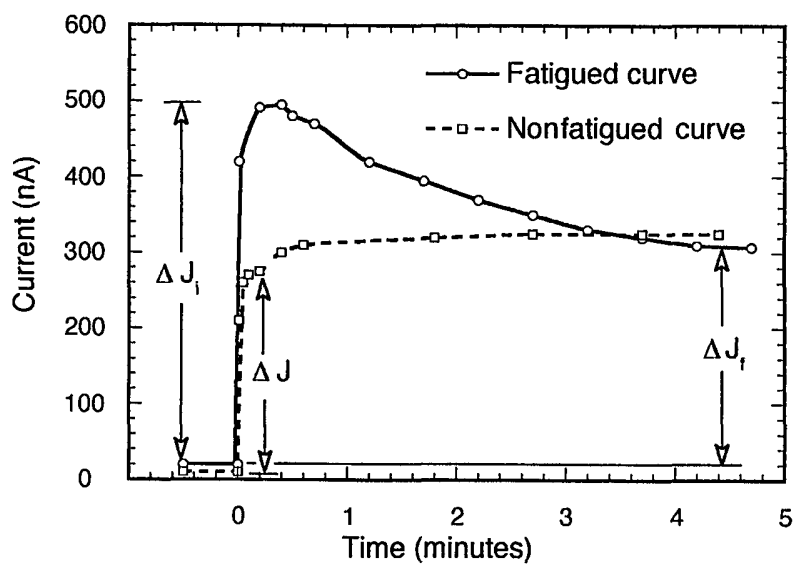

Fig. 6. Circles, fatigue of the photocurrent in sample 4, 20 days after fabrication; $\Delta J_{i}$ and $\Delta J_{f}$ are initial and final values of the photocurrent, respectively. Squares, nonfatigued photocurrent of sample 5, 21 days after fabrication. Both curves were recorded at $69 \mathrm{kV} / \mathrm{cm}, 650 \mathrm{~nm}$, and $50 \mathrm{~mW}$.

Table 1. Initial Photosensitivities of bisA-NAS:40-wt. \% DEH Samples ${ }^{a}$

\begin{tabular}{ccc}
\hline Sample & $\begin{array}{c}\text { Photosensitivity }\left(S_{\mathrm{ph}}\right) \\
(\mathrm{cm} / \mathrm{W} \Omega)\end{array}$ & $\begin{array}{c}\text { Time since } \\
\text { Fabrication }(\mathrm{h})\end{array}$ \\
\hline 1 & $1.31 \times 10^{-10}$ & 2 \\
2 & $0.51 \times 10^{-10}$ & 5.5 \\
3 & $0.40 \times 10^{-10}$ & 3 \\
4 & $1.10 \times 10^{-10}$ & 2 \\
5 & $2.10 \times 10^{-10}$ & 2 \\
\hline
\end{tabular}

${ }^{a}$ All samples exhibited nonfatigued photocurrent curves for these initial measurements. Measurements were made with $100 \mathrm{~mW}$ of $650-\mathrm{nm}$ wavelength illumination with an electric field of $69 \mathrm{kV} / \mathrm{cm}$. 


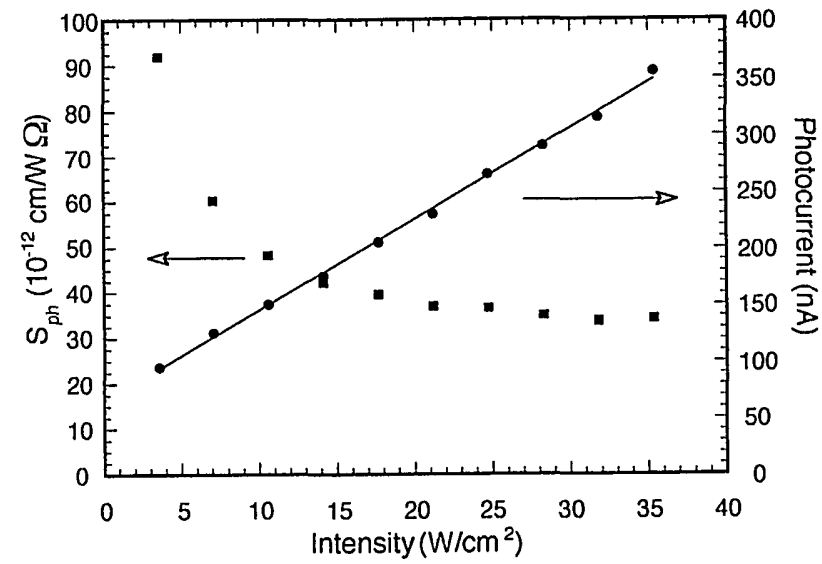

Fig. 7. Photocurrent (filled circles) and photosensitivity $S_{\mathrm{ph}}$ (filled squares) versus intensity in sample 1, recorded 3 days after fabrication. The photoconduction curves do not show fatigue.

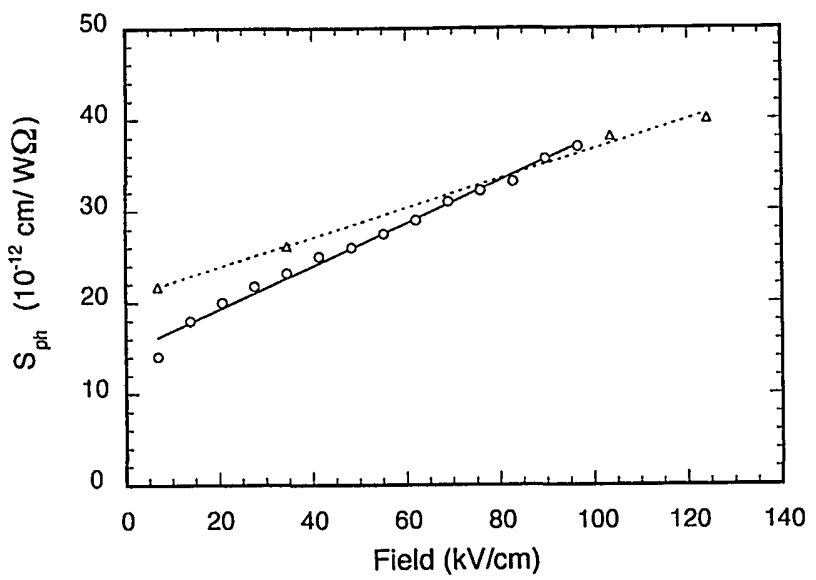

Fig. 8. Photosensitivity $S_{\text {ph }}$ versus field. Sample 3 (triangles) and sample 2 (circles) 1 day after fabrication at $650 \mathrm{~nm}$ and $100 \mathrm{~mW}$ power. The photoconduction curves do not show fatigue.

within the polymer host. ${ }^{3}$ A high concentration of DEH $(20-40 \mathrm{wt} . \%)$ is needed to produce sufficiently high photoconductivity; such concentrations presumably exceeded the room-temperature solubility of $\mathrm{DEH}$ in the polymer. Since the DEH functions as a conduit for charge carriers, a heterogeneous distribution of the molecule caused by precipitation results in low overall chargecarrier mobility. The fact that both the dark conductivity and the photosensitivity decreased with time indicated that it was the charge mobility, and therefore probably the $\mathrm{DEH}$, that was being affected, ruling out any explanation that would affect only the photosensitivity but not the dark conductivity, such as decreasing quantum efficiency or photogeneration rate. Since it was expected that the DEH would eventually clump together and form crystalline particles, $\mathrm{x}$-ray diffraction spectra were taken of month-old and 14-month-old bisA-NAS:DEH samples with a Rigaku $x$-ray diffraction spectrometer. These were then compared with an x-ray diffraction spectrum of powdered DEH crystals (Fig. 10). The 14-month-old sample showed several peaks that are characteristic of the DEH crystal spectrum, while samples 1 month or less in age showed no such peaks. Presumably the DEH in newer samples had not yet precipitated to the point at which it formed crystals big enough to be seen in x-ray spectra yet big enough to decrease the photosensitivity and the photorefractive speed significantly (Fig. 11).

\section{Photorefractive Measurements}

Photorefractive properties of the polymers were measured in degenerate four-wave mixing experiments with a cw dye laser operating at a wavelength of $650 \mathrm{~nm}$ with a single longitudinal mode and a $\mathrm{TEM}_{00}(1.0 \pm 0.05 \mathrm{~mm}$ FWHM) transverse mode. The experimental setup is shown in Fig. 12, and a close-up of the beam geometry near the sample is shown in Fig. 13. The sample was held on a stress-free mount at ambient temperature $\left(\approx 23{ }^{\circ} \mathrm{C}\right)$. The two $s$-polarized 20 - and $16.4-\mathrm{mW}$ writing beams were incident upon the sample at angles $\alpha=60^{\circ}$ and $\alpha-2 \theta=40^{\circ}$ (in air), respectively, to form a phase grating with wave vector $\mathbf{k}_{g}$. The angles $\alpha=60^{\circ}$ and $2 \theta=20^{\circ}$ result in a grating spacing of $2.6 \mu \mathrm{m}$ at an angle of $63.6^{\circ}$ from sample normal (assuming the sample refractive index $n \approx 1.7$ ). The $200 \mu \mathrm{W} p$-polarized reading beam 3 was incident counterpropagating to the object beam 2 and had a measurable effect only on the dark decay. Part of the reading beam was Bragg diffracted in

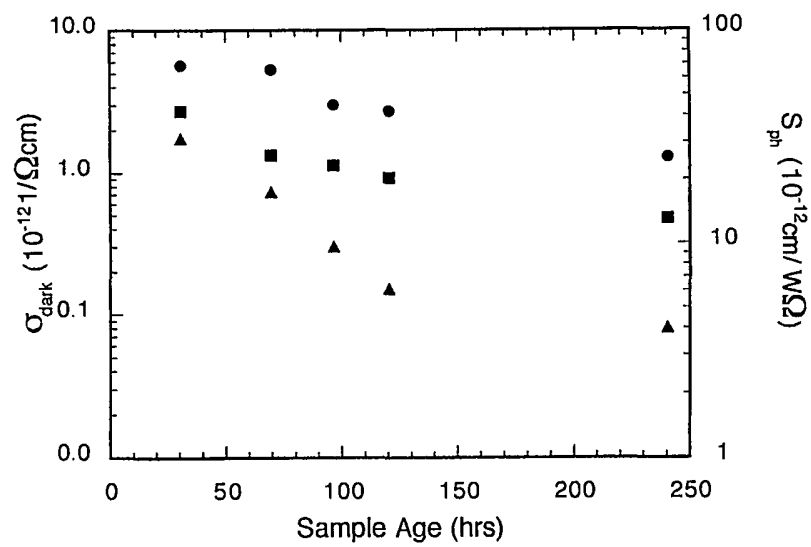

Fig. 9. Degradation of the initial photosensitivity (filled circles), the final photosensitivity (filled squares), and the dark conductivity $\sigma_{\text {dark }}$ (filled triangles) of sample 4 , which exhibits a fatigued photocurrent curve, with initial and final values of the photosensitivity $S_{\mathrm{ph}}$ corresponding to $\Delta J_{i}$ and $\Delta J_{f}$ as shown in Fig. 6.

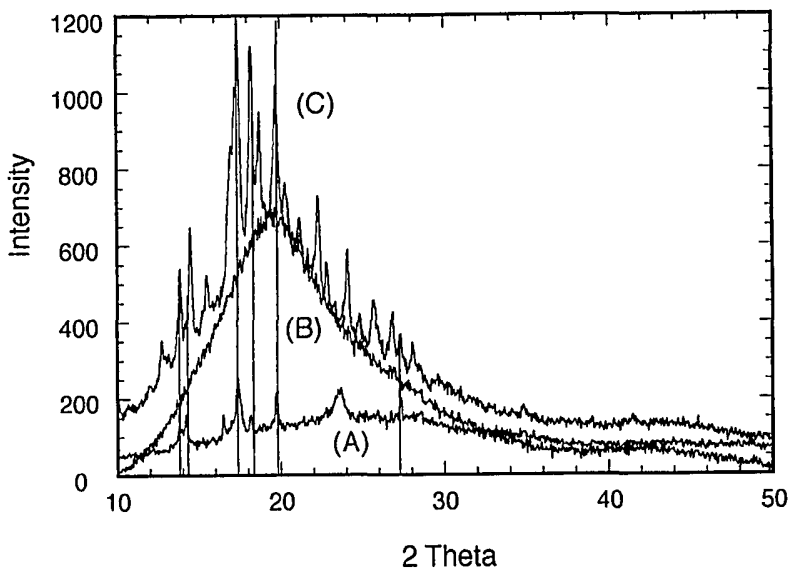

Fig. 10. X-ray diffraction spectra of powdered DEH (A) and of bisA-NAS: 40 wt. $\%$ DEH samples 1 month after fabrication (B) and 14 months after fabrication (C). 


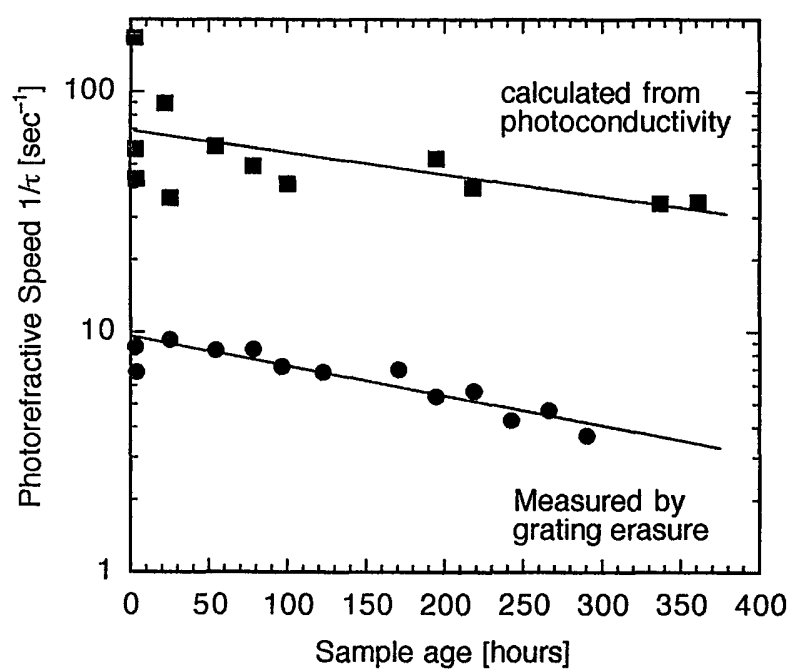

Fig. 11. Photorefractive speed $(1 / \tau)$ as measured in four-wave mixing (filled circles) and as calculated from photoconductivity measurements (filled squares) versus age of sample 5 at $650 \mathrm{~nm}$ and $20 \mathrm{~mW}$. The solid lines are guides to the eye.

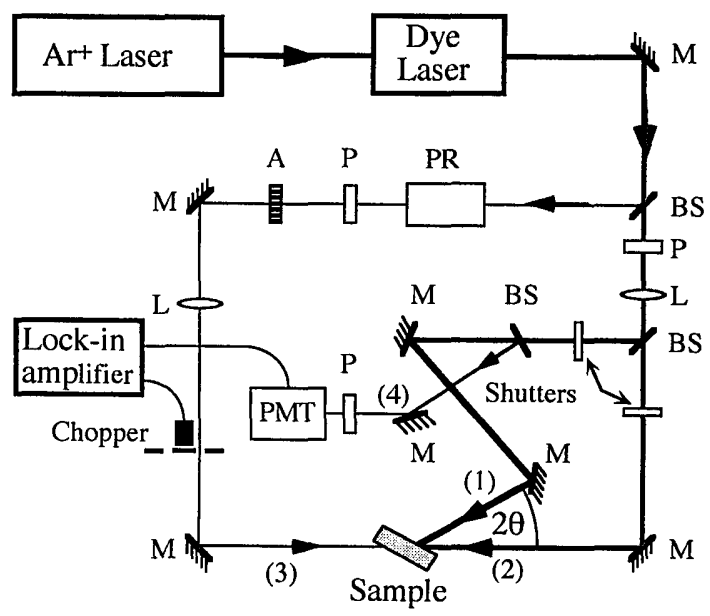

Fig. 12. Experimental setup of four-wave mixing measurements: L's, lenses; M's, mirrors; BS's, beam splitters; P's, polarizers; $\mathrm{PR}$, polarization rotator; $\mathrm{A}$, attenuator; $\mathrm{PMT}$, photomultiplier tube. The wavelength of the dye laser output was $650 \mathrm{~nm}$. The writing beams are (1) and (2), the reading beam is (3), and the signal beam is (4).

the direction opposite to beam 1 , and a beam splitter was used to direct the signal beam into a photomultiplier tube. Note that the small birefringence of the sample causes the oppositely polarized reading and writing beams to refract differently at the sample surface, so that the reading beam should not exactly counterpropagate to beam 2 for optimum diffraction. In practice this condition is satisfied by adjustment of the reading beam for maximum diffracted signal. The polarization of the reading beam and thus of the signal beam was orthogonal to that of the writing beams, so that the polarizer placed in front of the photomultiplier tube blocked most of the light scattered from beams 1 and 2 by sample inhomogeneities, optical components, and dust. The polarizer therefore improved the signal-to-noise ratio. The readout beam was $p$-polarized, since the larger electro-optic coefficient for this polarization produced a higher diffraction efficiency. The signalto-noise ratio was further improved by chopping of the reading beam at $2000 \mathrm{~Hz}$ and use of a lock-in amplifier to amplify the signal.

The photorefractive nature of the gratings was confirmed by observation of asymmetric two-beam energy coupling, which requires the presence of a shifted phase grating. ${ }^{12,23}$ The Bragg nature of the diffraction in fourwave mixing was verified by rotation of the sample during readout. The signal was observed during readout in unpoled samples only with the bias electric field present; there was no linear electro-optic response in zero field and hence no linear index grating, even in the presence of a photorefractive space-charge distribution. The holographic signal was large enough to measure only when a large electric field was applied to the sample during grating formation, consistent with field-enhanced (drift) photorefractive grating formation. ${ }^{2,3}$ The gratings could be repeatedly written and completely erased with the same optical wavelength and at fixed temperature. Two significant features of photorefractive polymers, as contrasted with crystals, are the large observed space-charge electric field resulting from the small dielectric constant and one's ability to turn the hologram diffraction on and off by repeatedly removing and reapplying the electric field $E_{0}$ after the space charge has been stored. ${ }^{3}$ The maximum observed diffraction efficiency in sample 1 before poling was $7.5 \times 10^{-5}$ at a bias field of $69 \mathrm{kV} / \mathrm{cm}$. The photorefractive diffraction efficiency in sample 1 was $1 \times 10^{-6}$ after poling, much smaller than before poling, perhaps because of the damage to the sample. The measurements reported below were made on unpoled samples; readout was possible only when an external electric field was applied.

The unpoled samples required an applied electric field to align the nonlinear-optical molecules and thus produce electro-optic response, as noted above. Usually $1000 \mathrm{~V}$ was applied to the $145-\mu \mathrm{m}$-thick samples, producing an electric-field strength of $69.0 \mathrm{kV} / \mathrm{cm}$. The diffraction efficiency, defined as the ratio of the diffracted power to the transmitted power, was recorded by a computer that also opened and closed the shutters in beams 1 and 2 at appropriate times. Dark decay curves were recorded with both beams 1 and 2 off, and light-induced erasure with only beam 2 on (though the weak beam 3 was always on). Usually five curves per measurement were recorded and analyzed separately, after which the decay times were

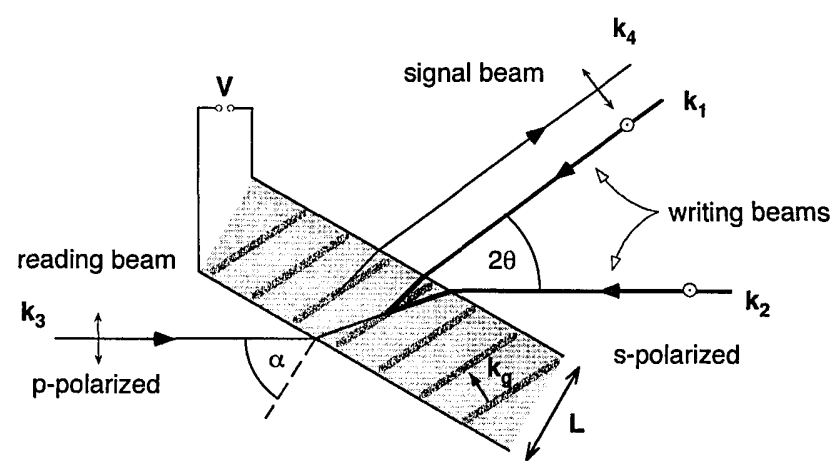

Fig. 13. Close-up schematic of light beams near the sample: $\mathbf{k}_{1}, \ldots, \mathbf{k}_{4}$, wave vectors of the four beams; $\mathbf{k}_{g}$, grating wave vector; $V$, applied voltage; $L$, sample thickness. The signal beam, $\mathbf{k}_{4}$, is shown displaced from the writing beam, $\mathbf{k}_{1}$, for clarity. 


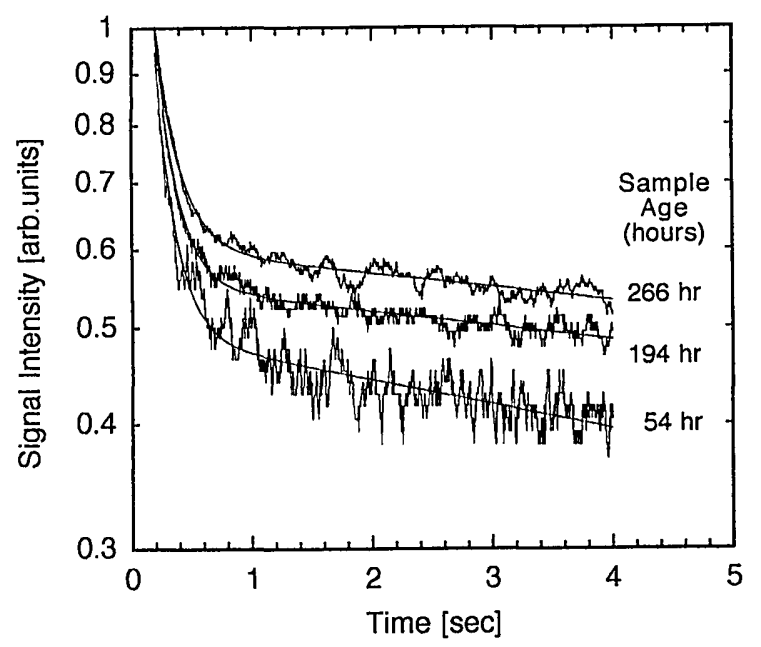

Fig. 14. Semilog plot of the example decay curves at three different sample ages. The solid curves are fits to the data as described in the text.

averaged. The erasure curves (see Fig. 14) were analyzed by an empirical two-exponential fit of the following form to the data:

$$
I=[a \exp (-t / b)+c \exp (-t / d)]^{2},
$$

where $a, \ldots, d$ are the four fitting parameters. The reason for choosing this particular fit can be most easily seen in Fig. 14, where example curves at three different times are plotted on a semilog scale. As can be seen in this figure, the data can be approximated by straight lines initially and at later times. Therefore it seems that the signal is best described by the two exponentials, whereas a single-exponential decay would appear as a single straight line in Fig. 14. Note, though, that qualitative conclusions drawn from Fig. 11 are not dependent on the precise form of the assumed decay curve [Eq. (1)]. In general it was observed that the short time constant was $\sim 100$ times smaller than the long time constant. For comparison of grating decay and photoconductivity, the short time constant was used. The exponential decay time of the grating amplitude was $\tau=0.11 \pm 0.02 \mathrm{~s}$ at intensity $I=1.0 \mathrm{~W} / \mathrm{cm}^{2}$, whereas the expected time constant from the photoconductivity measurements is much smaller.

The speed of photorefractive grating formation or erasure is the inverse of the exponential time constant $\tau$ and benefits from the inherently low dielectric constant $\epsilon_{r}$ of polymers. $^{2}$ The speed $1 / \tau \approx \sigma_{\mathrm{ph}} / \epsilon_{r} \epsilon_{0}$ (in the largegrating-spacing limit) is proportional to the material photoconductivity $\sigma_{\mathrm{ph}}=S_{\mathrm{ph}} I$, where the photosensitivity $S_{\mathrm{ph}}$ is proportional to the carrier photogeneration rate, mobility, and lifetime.

The photorefractive speed of bisA-NAS:DEH $40 \%$ $\left(0.11 \mathrm{~s}\right.$ at $\left.1 \mathrm{~W} / \mathrm{cm}^{2}\right)$ is approximately 3500 times greater than the first photorefractive polymer bisA-NPDA:DEH $30 \%\left(\tau=390 \mathrm{~s} \text { at } 1 \mathrm{~W} / \mathrm{cm}^{2}\right)^{3}$ The 40 -times-larger absorption coefficient of the NAS polymer would provide a 10.fold increase in speed, and the data in Ref. 3 project that a bisA-NPDA:DEH $40 \%$ mixture would be 5-10 times faster than the samples with $30 \% \mathrm{DEH}$. Together these factors account for an improvement of photosensitivity or photorefractive speed of $200-400$, much less than the observed improvement. The NAS chromophore has a greater conjugation length than the 4-nitro-1,2phenylenediamine (NPDA) chromophore and therefore will have larger separation of charge in the exited state and a higher quantum efficiency in transferring free holes to $\mathrm{DEH}$, perhaps explaining the higher photosensitivity.

Table 2 lists the photorefractive performance of several polymers and an organic crystal under comparable conditions, all without added photosensitizers. The photorefractive sensitivity $\mathrm{PRS}=\Delta n / \alpha I$, or photorefractive index change per absorbed energy density, is a combined measure of the electro-optic response, photogeneration quantum efficiency, and mobility-lifetime product, but does not depend on the absorption coefficient. The photorefractive sensitivity depends on experimental conditions such as wavelength, temperature, sample orientation, and polarization. In addition, the photorefractive sensitivity is noticeably increased by doping or sensitization, as has been already accomplished in several photorefractive polymers. ${ }^{14,25}$

\section{E. Relationship between Photoconductivity and Photorefractive Speed}

The standard model of photorefractive charge transport ${ }^{2}$ assumes the existence of partially filled (type A) traps; charge carriers are generated at one location and retrapped at another, producing space-charge distributions. Oppositely charged compensating traps, possibly defects or impurities, are postulated to satisfy overall charge neutrality. The compensating traps are assumed to be inert; they do not contribute to charge transport or to space charge. There is reason to believe $e^{3,12,26}$ that the standard model is applicable to photorefractive polymers. Specific features of conduction in, e.g., geminate recombination, ${ }^{21,27,28}$ low mobility, ${ }^{27,29}$ and large carrier range, can be readily incorporated into the standard model. ${ }^{27,28}$

As is discussed above, the observed photoconductive fatigue in the photorefractive polymers is consistent with the standard model if we postulate the presence of additional shallow type $B$ traps. The same shallow type $B$ traps can store space charge in a competing, antiphase grating in competition with the usual grating stored in type A traps. This model has been applied to observations of grating competition in photorefractive crystals $^{30-33}$ and in a photorefractive polymer. ${ }^{6}$ We now have three types of fixed charge in this model, the photoionizable type A traps that are the source of photocarriers, the completely immobile compensating charge, and the nonphotosensitive type $B$ traps that trap free carriers and are emptied by thermal excitation only. The model explains fatigue of the photoconductivity, the slow recovery of this fatigue, and photorefractive grating competition. Only the type A traps have an identifiable source, the NAS molecules. The most compelling evidence for the presence of the type B traps in the bisANAS:DEH polymers is the photoconduction fatigue. The grating competition observed in bisA-NAT is also consistent with the presence of type $B$ traps.

Photorefractive theory predicts that, to a first approximation with the contributions of the type B traps neglected, the photorefractive speed of grating formation or erasure $1 / \tau$ is proportional to the photosensitivity $S_{\mathrm{ph}}$. 
Table 2. Diffraction Efficiency $\eta$, Absorption Coefficient $\alpha$, and Photorefractive Sensitivity PRS of Some Photorefractive Polymers (without Additional Sensitizers) and an Organic Photorefractive Crystal (with Sensitizer) ${ }^{a}$

\begin{tabular}{lcccrr}
\hline \multicolumn{1}{c}{ Polymer } & $\tau$ at $1 \mathrm{~W} / \mathrm{cm}^{2}(\mathrm{~s})$ & $\eta$ & $\alpha\left(\mathrm{cm}^{-1}\right)$ & Bias Field $(\mathrm{V} / \mathrm{cm})$ & ${\text { PRS }\left(\mathrm{cm}^{3} / \mathrm{J}\right)}$ \\
\hline BisA-NPDA $^{b}$ & 390 & $0.9 \times 10^{-4}$ & 1.5 & 126 & $1 \times 10^{-8}$ \\
PMMA-PNA $^{c}$ & 6.7 & $7.5 \times 10^{-6}$ & 0.57 & 114 & $3.9 \times 10^{-7}$ \\
BisA-NAT $^{d}$ & 1.1 & $1.1 \times 10^{-3}$ & 118 & 138 & $3.4 \times 10^{-7}$ \\
BisA-NAS $^{\text {MNBA crystal }}$ & 0.11 & $7.5 \times 10^{-5}$ & 60 & 138 & 0 \\
\hline
\end{tabular}

${ }^{a}$ Measurements were made near the 650 -nm wavelength, with $p$-polarized readout and with $1-2 \mu \mathrm{m}$ grating spacing, unless otherwise noted.

${ }^{b}$ Ref. 3.

${ }^{c}$ Ref. 5 .

${ }^{d}$ Ref. 6.

${ }^{e}$ Ref. 38 , measured at $676 \mathrm{~nm}$.

However, it was found that the time constant calculated from photoconductivity measurements did not agree with the actual time necessary to form a grating in four-wave mixing experiments. The actual time necessary to form a grating was $10-800$ times greater than the predicted time. This may mean that there are parallel photoconduction channels (i.e., additional traps) that contribute to photoconduction but not to photorefractive charge storage. This question deserves further study.

To examine the relationship between the photorefractive response time and the photosensitivity, a sample was monitored over a time of just under $400 \mathrm{~h}$. The photosensitivity of the sample decreased over this time, presumably because of $\mathrm{DEH}$ precipitation as discussed above. The photorefractive speed decreased in proportion to the photosensitivity, as is shown in Fig. 11.

\section{F. Two-Beam Energy Coupling}

Two-beam energy coupling measurements in a BisANAS:DEH sample made with extraordinary polarization, with $30-\mathrm{mW}$ pump- and $3.9-\mathrm{mW}$ probe-beam powers, yielded $g L=0.74 \pm 0.1 \%$ as the coupling strength, or $g=0.46 \pm 0.06 \mathrm{~cm}^{-1}$ as the coupling gain coefficient, with an applied field of $138 \mathrm{kV} / \mathrm{cm}$ and grating orientation and spacing as above. With the grating phase assumed to be $90^{\circ}$, the coupling strength should be $g L=2 \sqrt{\eta}$, where $\eta$ is the degenerate four-wave mixing diffraction efficiency. A value of $\eta=0.0075 \pm 0.002 \%$ measured with the same sample under the same conditions yielded a calculated coupling strength of $1.7 \%$, larger than the measured coupling of $0.74 \%$, perhaps owing to the observed inhomogeneity of the electro-optic response, although it is also consistent with a grating phase different from $90^{\circ} .4$

\section{CONCLUSIONS}

While these first-generation photorefractive polymer mixtures represent a breakthrough in the design of new organic photorefractive materials, and there is much to be learned about their properties, they will ultimately fail to satisfy some essential requirements for practical photorefractive materials. One of these failures is inherent in the need for large amounts of the charge transport agent, such as DEH, far above its solubility in the host polymers. This approach inevitably leads to polymer mixtures with a low $T_{g}$ and with optical inhomogeneity, low dielectric strength, phase separation, and degradation of the photosensitivity.
We have monitored the simultaneous degradation of the photosensitivity, the dark conductivity, and the photorefractive speed of samples of BisA-NAS:DEH. ${ }^{34}$ This degradation, also observed in bisA-NAT:DEH (Ref. 6) and bisA-NPDA:DEH, ${ }^{3}$ is likely due to precipitation of the $\mathrm{DEH}$, since $\mathrm{DEH}$ is not soluble at the large concentrations ( $20-40 \mathrm{wt} . \%)$ necessary to produce significant photoconduction in these polymers. Covalent attachment of the charge-transport agent is an appropriate alternative approach that will eliminate precipitation and consequently eliminate the degradation of the photoconductivity and increase the glass-transition temperature. Such an approach has been demonstrated. ${ }^{35}$ Advances in high-temperature stability of nonlinearoptical polymers ${ }^{16,17,36,37}$ should then be generally applicable to these covalent photorefractive polymers.

Comparisons of the absorption coefficient, photosensitivity, and photorefractive speed have established that the nonlinear-optical chromophore also serves as the photosensitizer in the bisA-NPDA:DEH, ${ }^{3}$ bisA-NAS:DEH, ${ }^{34}$ and bisA-NAT:DEH systems. ${ }^{6}$ Improvement of the photoconductivity, and also of the speed, of photorefractive polymers by photosensitization with dyes (including fullerenes) has been reported by several groups. ${ }^{5,7,14,24}$ Sensitization with appropriate dyes is a versatile means of improving diffraction efficiency and speed at technologically important semiconductor laser wavelengths.

These studies are guiding synthesis of new polymers, particularly polymers with covalently attached charge, transport agents that do not phase separate and have higher $T_{g}$, higher dielectric strength, improved homogeneity, and stable photoconductivity. We have qualitative correspondence between photoconductivity and photorefractive speed. However, the photorefractive speed is consistently smaller than is predicted by photorefractive theory from the measured photoconductivity of the samples. Future studies should concentrate on photorefractive characterization of the new polymers to test the applicability of theoretical models. ${ }^{2,27,28}$

\section{ACKNOWLEDGMENTS}

Sam Rankin measured the dielectric constants and Rich Ervin poled two bisA-NAS:DEH samples. This work was supported by a Research Initiation Award from the National Science Foundation Directorate for Engineering (ECS-9209941), by the Nebraska Research Initiative through the Center for Materials Research and Analy- 
sis, and by the Research Council of the University of Nebraska.

\section{REFERENCES}

1. A. Ashkin, G. D. Boyd, J. M. Dziedzic, R. G. Smith, A. A. Ballman, J. J. Levinstein, and K. Nassau, Appl. Phys. Lett. 9, 72 (1966).

2. N. V. Kukhtarev, V. B. Markov, S. G. Odulov, M. S. Soskin, and V. L. Vinetskii, Ferroelectrics, 22, 949 (1979).

3. S. Ducharme, R. W. Twieg, J. C. Scott, and W. E. Moerner, Phys. Rev. Lett. 66, 1846 (1991).

4. C. A. Walsh and W. E. Moerner, J. Opt. Soc. Am. B 9, 1642 (1992).

5. S. M. Silence, C. A. Walsh, J. C. Scott, T. J. Matray, R. J. Twieg, F. Hache, G. C. Bjorklund, and W. E. Moerner, Opt. Lett. 17, 1107 (1992).

6. S. Ducharme, B. Jones, J. M. Takacs, and L. Zhang, Opt. Lett. 18, 152 (1993).

7. Y. Zhang, Y. Cui, and P. N. Prasad, Phys. Rev. B. 46, 9900 (1992).

8. D. S. Chemla and J. Zyss, ed., Nonlinear Optical Properties of Organic Molecules and Crystals (Academic, Orlando, Fla., 1987), Vol. 1.

9. R. A. Hann and D. Bloor, ed., Organic Materials for Nonlinear Optics (Royal Society of Chemistry, London, 1989).

10. M. Lardon, E. Lell-Döeller, and J. W. Weigl, Mol. Cryst. 2, 241 (1967).

11. A. S. Diamond, Handbook of Imaging Materials (Marcel Dekker, New York, 1991).

12. M. Liphardt, A. Goonesekera, B. E. Jones, S. Ducharme, J. M. Takacs, and L. Zhang, Science 263, 367 (1994).

13. S. Ducharme, A. Goonesekera, B. Jones, J. M. Takacs, and L. Zhang, in Organic Thin Films for Photonic Applications, Vol. 17 of 1993 Technical Digest Series, G. C. Bjorklund, A. D. English, C. W. Frank, and K. D. Singer, eds. (Optical Society of America, Washington, D.C., 1993), p. 232.

14. S. M. Silence, C. A. Walsh, J. C. Scott, and W. E. Moerner, Appl. Phys. Lett. 61, 2967 (1992).

15. M. C. J. M. Donckers, S. M. Silence, C. A. Walsh, F. Hache, D. M. Burland, W. E. Moerner, and R. J. Twieg, Opt. Lett. 18, 1044 (1993).

16. J. W. Wu, J. F. Valley, S. Ermer, E. S. Binkley, J. T. Kenner, G. F. Lipscomb, and R. Lytel, Appl. Phys. Lett. 58, 225 (1991).

17. M. Eich, B. Reck, D. Y. Yoon, C. G. Willson, and G. C. Bjorklund, J. Appl. Phys. 66, 3241 (1989).
18. R. D. Miller, K. M. Betterton, D. M. Burland, V. Y. Lee, C. R. Moylan, R. J. Twieg, C. A. Walsh, and W. Volksen, in Organic Thin Films for Photonic Applications, Vol. 17 of 1993 OSA Technical Digest Series, G. C. Bjorklund, A. D. English, C. W. Frank, and K. D. Singer, eds. (Optical Society of America, Washington, D.C., 1993), p. 54.

19. S. Ducharme and J. Feinberg, IEEE J. Quantum Electron QE-23, 2116 (1987).

20. M. Eich, G. C. Bjorklund, and D. Y. Yoon, Polym. Adv. Technol. 1, 189 (1990).

21. L. Onsager, Phys. Rev. 54, 554 (1938)

22. J. X. Mack, L. B. Schein, and A. Peled, Phys. Rev. B 39, 7500 (1989).

23. C. A. Walsh D. M. Burland, V. Y. Lee, R. D. Miller, B. A. Smith, R. J. Twieg, and W. Volksen, Macromolecules 26, 3720 (1993).

24. Y. Cui, Y. Zhang, P. N. Prasad, J. S. Schildkraut, and D. J. Williams, Appl. Phys. Lett. 61, 2132 (1992).

25. W. E. Moerner and S. Silence, Chem. Rev. 94, 127 (1994).

26. J. C. Scott, S. Ducharme, R. J. Twieg, and W. E. Moerner, A.C.S. Polymer Preprints 32, 107 (1991).

27. A. Twarowski, J. Appl. Phys. 65, 2833 (1989).

28. J. S. Schildkraut and A. V. Buettner, J. Appl. Phys. 72, 1888 (1991).

29. J. C. Scott, L. T. Pautmeier, and W. E. Moerner, J. Opt. Soc. Am. B 9, 2059 (1992).

30. D. D. Nolte, D. H. Ohlson, and A. M. Glass, Phys. Rev. Lett. 63, 891 (1989)

31. M. C. Bashaw, T. P. Ma, R. C. Barker, S. Mroczkowski, and R. R. Dube, Phys. Rev. B 42, 5641 (1990).

32. P. Tayebati and D. Mahgerefteh, J. Opt. Soc. Am. B 8, 1053 (1991).

33. D. Kirillov and J. Feinberg, Opt. Lett. 16, 1520 (1991).

34. S. Ducharme, B. Jones, M. Liphardt, R. Ervin, J. M. Takacs, L. Zhang, and R. Athalye, in Proceedings of the Topical Meeting on Photorefractive Materials, Effects, and Devices, B. Y. Zel'dovich and E. Krätzig, eds. (Ukrainian Academy of Sciences, Kiev, 1993), p. 1.

35. K. Tamura, A. B. Padias, J. H. K. Hall, and N. Pehghambarian, Appl. Phys. Lett. 60, 1803 (1992).

36. B. Reck, M. Eich, D. Jungbauer, R. Twieg, C. G. Willson, D. Y. Yoon, and G. C. Bjorklund, in Nonlinear Optical Properties of Organic Materials II, G. Khanarian, ed., Proc. Soc. Photo-Opt. Instrum. Eng. 1147, 2610 (1989).

37. D. Jungbauer, B. Reck, R. Twieg, D. Y. Yoon, C. G. Willson, and J. D. Swalen, Appl. Phys. Lett. 56, 2610 (1990).

38. K. Sutter and P. Günter, J. Opt. Soc. Am. B 7, 2274 (1990). 